C-A/AP/\#251

August 2006

NSRL Energy Loss Calculator

B. VanKuik, C. Gardner, S. Bellavia, A. Rusek, K. Brown

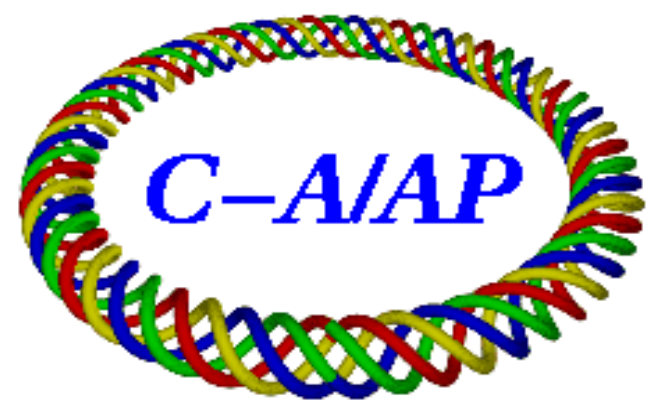

Collider-Accelerator Department Brookhaven National Laboratory

Upton, NY 11973 


\section{NSRL Energy Loss Calculator}

A means of calculating the energy lost down the NSRL beam line was needed. The program takes the geometry of the NSRL beam line, starting at the D6 foil drive, to the first Ion chamber that A. Rusek uses for the Bragg Curve measurements. Below is a description of how the NSRL Energy Loss Calculator works. The variable definitions are similar to those variable names used in the program. Note that the beam energy quoted in the NSRL Target Room is just before entering the first ion chamber that makes up the Bragg Curve Equipment (see appendix C).

Access to program through a Linux terminal window:

1.) At prompt $>$ cd $/$ home/cfsb/kuik/Programs/Tcl_Tk/NSRL

2.) At prompt>xemacs NsrlEnergyLossInteractive.tcl \& (to view the source code)

3.) At prompt $>$ NsrlEnergyLossInteractive.tcl \& (to run the program)

Variable definitions:

$\mathrm{TMZ}=$ Target Material Atomic Number

TMA $=$ Target Material Atomic Mass

TMT $=$ Target Material Thickness (in $\mathrm{cm}$ converted from inches)

$\mathrm{TMD}=$ Target Material Density $\left(\mathrm{g} / \mathrm{cm}^{\wedge} 3\right)$

TMI = Target Material Mean Excitation Energy $(\mathrm{eV})$

BeamZ $=$ Charge of the Beam

Beam_n = Number of Nucleons per ion for the Beam rme $=$ Rest Mass Energy of the beam $(\mathrm{GeV})$

I. Set the Beam type (Protons, Iron, Copper, Titanium, Carbon, etc...)

1.) Set the beam's Charge, Atomic Weight, Rest Mass Energy, \& Nucleon Count (z, beam_a,rme, beam_n)

II. Set Starting Kinetic Energy as measured at Booster Extraction

1.) Measure the RF Frequency (freqRF) at Booster Extraction $(\mathrm{MHz})$

2.) Using nominal Booster radius

$$
\text { boosterR }=32.11428 \text { meters }
$$

3.) Convert frequency from $\mathrm{MHz}$ to $\mathrm{Hz}$

$$
\text { freqRF }=\text { freq } R F * 10^{6}
$$

4.) Convert Rest Mass Energy of the Beam (rme) from $\mathrm{GeV}$ to $\mathrm{MeV}$

$$
r m e=r m e^{*} 10^{3}
$$

5.) Measure the radial shift dr at Booster Extraction in millimeters.

6.) Convert radial shift from millimeters to meters

$$
d r=\frac{d r}{10^{3}}
$$

7.) Adjust the booster extraction radius

$$
\text { booster } R=\text { booster } R+d r
$$

8.) Calculate Beta, where $h$ is the harmonic number and $c$ is the speed of light:

$$
\beta=\frac{2 * \pi * \text { booster }{ }^{*} \text { freqRF }}{h^{*} c}
$$

9.) Calculate Gamma

$$
\gamma=\frac{1}{\sqrt{1-\beta^{2}}}
$$


10.) Calculate total energy (MeV/ion)

$$
\text { energyTot }=\gamma * \text { rme }
$$

11.) Calculate kinetic energy per ion (MeV/ion)

$$
\text { boosterExtKE }=\text { energyTot }- \text { rme }
$$

12.) Convert kinetic energy per ion to energy per nucleon $(\mathrm{MeV} / \mathrm{n})$

$$
\text { boosterExtKE }=\frac{\text { boosterExtKE }}{\text { Beam_n }_{-}}
$$

III. Intercepting the various materials.

Starting with the Booster Extracted Kinetic Energy this value gets put into two variables previousKE \& newKE. The first variable, previous $K E$, is used to calculate the overall energy loss if the intercepting material is composed of several types of material. The second variable, newKE, is the energy of the beam intercepting the material. The energy of the beam always becomes the newKE being indifferent to either a slice of material or a new material intercepting the beam.

The next several steps are basic to every material the beam intercepts in the NSRL beam line.

1.) Get the specific values for the material the beam is intercepting

a.) set the target material

b.) set the target thickness (convert to centimeter from inches)

c.) set the $Z$ (atomic number), $A$ (atomic mass), Z/A (ratio of the atomic number over the atomic weight), TMI (mean excitation energy), \& density for the target material

i.) Calculate $\frac{Z}{A}$ if it has not been provide for in the tables.

ii.) Calculate the Mean Excitation Energy $T M I=16 * Z^{0.9}$ of the material if the experimental number is not available.

2.) Convert $\mathrm{TMI}$ from $\mathrm{eV}$ to $\mathrm{MeV}$ :

$$
T M I=T M I *\left(\frac{1}{10^{6}}\right)
$$

3.) Calculate gamma from Kinetic Energy:

$$
\gamma=\frac{n e w K E+r m e}{r m e}
$$

4.) Calculate Beta from Gamma:

$$
\beta=\sqrt{1-\frac{1}{\gamma^{2}}}
$$

5.) Calculate Bethe-Bloch ${ }^{[1]}[2] \mathrm{dE} / \mathrm{dx}$

$$
\begin{aligned}
-\frac{d E}{d x}=K * \operatorname{BeamZ}^{2} * \frac{T M Z}{T M A} * \frac{1}{\beta^{2}} *\left[0.5 * \ln \left(\frac{2.0 * m_{e} c^{2} * \beta^{2} * \gamma^{2} * n e w K E}{T M I^{2}}\right)-\beta^{2}\right] \\
K=0.3071 \mathrm{MeV} \\
m_{e} c^{2}=0.5109906 \mathrm{MeV}
\end{aligned}
$$

6.) Calculate $\mathrm{dE}$, in units of $\mathrm{MeV}$, by multiplying the Target Material's thickness and density to the Bethe-Bloch result.

$$
d E=\frac{d E}{d x} * T M T * T M D
$$


7.) Calculate new Kinetic Energy (newKE) in units of MeV per nucleon:

$$
n e w K E=\frac{n e w K E^{*} \text { Beam_n-dE }}{\text { Beam } n}
$$

8.) Move on to the next material and repeat up to the point that the air gap just before the plastic used for Bragg curve measurements.

The D6 flag drive is the first object the beam sees after leaving the Booster. There are 8 possible positions for the drive with one being a blank target. Afterward there are 4 insertable instrumentation packages in the NSRL beam line, an aluminum window, and a permanently inserted instrumentation package in the target room (see Appendix C). Each instrumentation package contains an ion chamber (made up of 5 aluminum planes), a SWIC (both horizontal and vertical measuring planes), and a scintillator detectors (see appendix D). When the insertable instrumentation packages are retracted, the beam only sees vacuum. The permanently inserted instrumentation package is flanked by air gaps on both sides. All of the instrumentation packages are filled with nitrogen gas.

Each material that the beam intercepts is sliced up into 40 parts to ensure that energy loss is close to as fully accounted for by the material. In some cases the material is thin enough where this is most likely overkill. Even though this is probably overkill, there is a mix of materials within the instrumentation packages with thicknesses varying from 0.001 ” to 0.780 ” thick. Also of note, for the D6 foil, those beam species whose charge is stripped full after the D6 foil, the ion is considered fully stripped after the first slice of the material layer.

\section{Available :}

NSRL beam line Instrumentation layout http://www.c-ad.bnl.gov/esfd/nsrl/operations/InstruLayoutNSRL.pdf Atomic Information used (see Appendix A)

Program Interface (see Appendix B)

NSRL target room layout (see Appendix C)

Instrument Package Cross Section (see Appendix D)

Send request for program code to B. van Kuik at vankuik@bnl.gov

\section{Acknowledgements:}

S. Bellavia \& A. Rusek for providing technical data on the NSRL beam line.

K. Brown, K. Gardner, \& A. Rusek for providing consultation. 


\section{Appendix A: Atomic Information}

Table of Element values used within the calculator. These data came from references [3], [4], and [5].

\begin{tabular}{|c|c|c|c|c|c|c|c|c|}
\hline Element & $\begin{array}{l}\text { Atomic } \\
\text { Symbol }\end{array}$ & $\mathbf{Z}$ & A & $\langle Z / A\rangle$ & $\begin{array}{c}\text { Mean } \\
\text { Excitation Energy } \\
e V \\
\mathrm{l}=16^{\star} \mathrm{Z}^{\wedge} 0.9\end{array}$ & $\begin{array}{c}\text { Experimental } \\
\text { Mean } \\
\text { Excitation Energy } \\
\text { eV }\end{array}$ & $\begin{array}{l}\text { Density } \\
\mathrm{g} / \mathrm{cm}^{\wedge} 3\end{array}$ & $\begin{array}{c}\text { Rest } \\
\text { Mass } \\
\text { GeV }\end{array}$ \\
\hline Hydrogen & $\mathrm{H}$ & 1 & 1.007825032 & 0.99212 & 16 & 19.2 & & 0.938272 \\
\hline Helium & $\mathrm{He}$ & 2 & 4.00260325 & 0.49968 & 29.85705573 & 41.8 & $1.66322 \mathrm{E}-04$ & \\
\hline Lithium & $\mathrm{Li}$ & 3 & 6.941 & 0.43221 & 43.00600607 & & & \\
\hline Beryllium & $\mathrm{Be}$ & 4 & 9.012182 & 0.44384 & 55.71523605 & 63.7 & 1.848 & 11.1754 \\
\hline Carbon & $\mathrm{C}$ & 6 & 12.00000 & 0.49954 & 80.252045 & 81.0 & 2.0 & \\
\hline Nitrogen & $\mathrm{N}$ & 7 & 14.00674 & 0.49976 & 92.19518045 & 82.0 & $1.16528 \mathrm{E}-03$ & \\
\hline Oxygen & To & 8 & 15.99491462 & 0.50002 & 103.9683067 & 95.0 & $1.33151 \mathrm{E}-03$ & 14.8956 \\
\hline Flourine & $F$ & 9 & 18.9984032 & 0.47372 & 115.5947849 & & & \\
\hline Aluminum & $\mid \mathrm{Al}$ & 13 & 26.981539 & 0.48181 & 160.9418539 & 166.0 & 2.6989 & \\
\hline Sillicon & $\mathrm{Si}$ & 14 & 27.97692653 & 0.49848 & 172.04229 & 173.0 & 2.33 & 26.0536 \\
\hline Chlorine & $\mathrm{Cl}$ & 17 & 35.453 & 0.49848 & 204.891533 & & & 32.5661 \\
\hline Argon & $\mathrm{Ar}$ & 18 & 39.948 & 0.45059 & 215.7074959 & 188.0 & $1.66201 \mathrm{E}-03$ & \\
\hline Titanium & $\mathrm{Ti}$ & 22 & 47.9479471 & 0.45948 & 258.4046922 & 233.0 & 4.54 & 44.65 \\
\hline Iron & $\mathrm{Fe}$ & 26 & 55.9349421 & 0.46556 & 300.3281188 & 286.0 & 7.874 & 52.09 \\
\hline Copper & $\mathrm{Cu}$ & 29 & 62.9296011 & 0.45636 & 331.3432905 & 322.0 & 8.96 & \\
\hline Tungsten & W & 74 & 183.84 & 0.40250 & 769.8897372 & 727.0 & 19.3 & \\
\hline Gold & $\mathrm{Au}$ & 79 & 196.966552 & 0.40108 & 816.5529738 & 790.0 & 19.32 & 183.457 \\
\hline Kapton & $\mathrm{nm}$ & 6.35993 & 12.70055 & 0.50076 & 84.57209418 & 79.6 & 1.42 & 0 \\
\hline Carbon Dioxide & $\mathrm{CO} 2$ & 7.454168 & 14.90698 & 0.50005 & 97.56167356 & 85.0 & $1.84 \mathrm{E}-03$ & \\
\hline Silicon Dioxide & $\mathrm{SiO} 2$ & 10.80461 & 21.64599 & 0.49915 & 136.2599322 & 139.2 & 2.32 & \\
\hline Air & $\mathrm{C}, \mathrm{N}, \mathrm{O}, \mathrm{Ar}$ & 7.372747 & 14.80005 & 0.49816 & 96.60205844 & \begin{tabular}{|l|}
85.7 \\
\end{tabular} & $1.20 \mathrm{E}-03$ & \\
\hline
\end{tabular}

Below are the computed values for Kapton, Carbon Dioxide, Silicon Dioxide, and Air. Each atomic numbers and atomic mass is multiplied by the weighting value for the material, then summed together to give the effective atomic numbers and atomic mass.

Compositional data provided by NIST Physics Laboratory website (http://physics.nist.gov/cgi-bin/Star/compos.pl?ap).

\begin{tabular}{|c|c|c|c|c|}
\hline \multicolumn{2}{|c|}{ Kapton Composition } & \multicolumn{2}{|c|}{ Weighting Weighted } & \multirow{2}{*}{$\begin{array}{c}\text { Weighted } \\
\text { A }\end{array}$} \\
\hline $\mathbf{Z}$ & A & Value & Z & \\
\hline 1 & 1.00794 & 0.026362 & 0.026362 & 0.026571314 \\
\hline 6 & 12.00000 & 0.691133 & 4.146798 & 8.293596 \\
\hline 7 & 14.00674 & 0.07327 & 0.51289 & 1.02627384 \\
\hline 8 & 15.994 & 0.209235 & 1.67388 & 3.34650459 \\
\hline & & effec & 6.35993 & 12.69294574 \\
\hline
\end{tabular}

\begin{tabular}{|c|c|c|c|c|}
\hline \multicolumn{2}{|l|}{$\mathrm{CO} 2$} & \multicolumn{2}{|c|}{ Weighting Weighted } & \multirow{2}{*}{$\begin{array}{c}\text { Weighted } \\
\text { A }\end{array}$} \\
\hline$\underline{Z}$ & A & Value & Z & \\
\hline 6 & 12.00000 & 0.272916 & 1.637496 & 3.274992 \\
\hline 8 & 15.994 & 0.727084 & 5.816672 & 11.6289815 \\
\hline & & effective & 7.454168 & 14.9039735 \\
\hline
\end{tabular}

\begin{tabular}{|c|c|c|c|c|}
\hline \multicolumn{2}{|l|}{$\mathrm{SiO} 2$} & \multicolumn{2}{|c|}{ Weighting Weighted } & \multirow{2}{*}{$\begin{array}{c}\text { Weighted } \\
\text { A }\end{array}$} \\
\hline $\mathbf{Z}$ & A & Value & $\mathbf{Z}$ & \\
\hline 8 & 15.994 & 0.532565 & 4.26052 & 8.51784461 \\
\hline 14 & 28.0855 & 0.467435 & 6.54409 & 13.12814569 \\
\hline & & effective & 10.80461 & 21.6459903 \\
\hline
\end{tabular}

\begin{tabular}{|c|c|c|c|r|}
\hline \multicolumn{4}{|l|}{ Air } & \multicolumn{4}{l|}{ Weighting Weighted } & \multicolumn{1}{c|}{ Weighted } \\
\hline $\mathbf{Z}$ & $\mathbf{A}$ & \multicolumn{1}{c|}{ Value } & \multicolumn{1}{c|}{ A } \\
\hline 6 & 12.00000 & 0.000124 & 0.000744 & 0.001488 \\
\hline 7 & 14.00674 & 0.755267 & 5.286869 & 10.5788285 \\
\hline 8 & 15.99491462 & 0.231781 & 1.854248 & 3.707317306 \\
\hline 18 & 39.948 & 0.012827 & 0.230886 & 0.512412996 \\
\hline \multirow{4}{*}{ effective } & 7.372747 & 14.800046802 \\
\hline
\end{tabular}


Below is the interface for the NSRL Energy Loss Calculator. To use the main function of calculating the energy loss down the NSRL beam line, enter a Beam Energy value in units of MeV into the Booster Extraction Energy field, select the beam species, and click the Calculate Energy Loss button. The program will then calculate the energy loss, retrieving any elements that are intercepting the beam in the live machine.

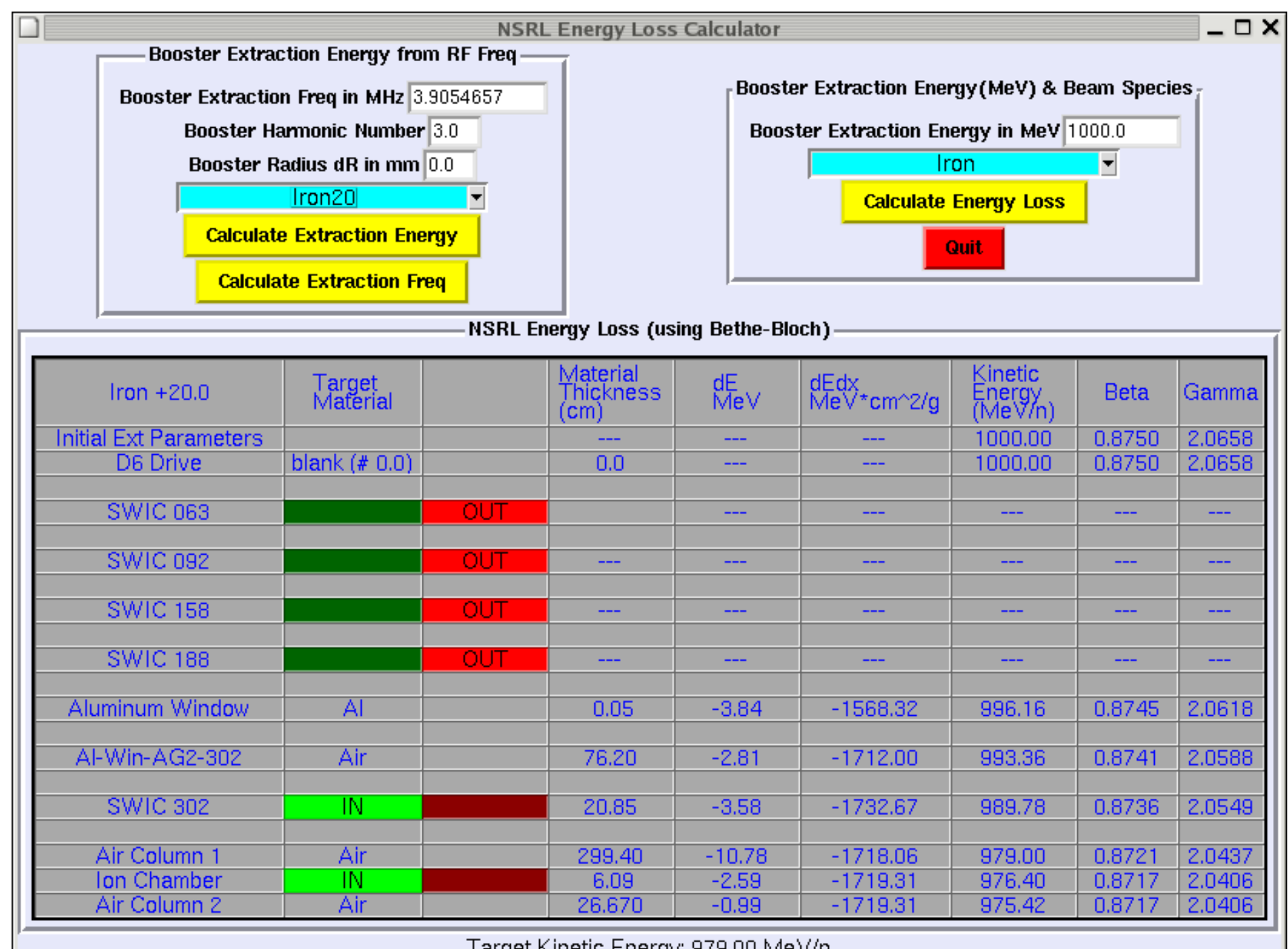

Target Kinetic Energy: $979.00 \mathrm{MeV} / \mathrm{n}$

There are two other functions contained in the NSRL Energy Loss Calculator the ability to calculate the Booster Extraction Energy from a measured extraction frequency and the ability to calculate the expected Booster Extraction Frequency for a given extraction energy. To calculate the Booster Extraction energy, enter the Booster Extraction frequency in $\mathrm{MHz}$ and the appropriate Booster harmonic number for the beam species. To calculate the Booster Extraction Frequency enter an extraction energy value in the Booster Extraction Energy field and enter the appropriate Booster harmonic number. Generally the harmonic numbers for protons and ions are $\mathrm{h}=2$ and $\mathrm{h}=3$ respectively. Click the relevant calculation button. The Booster Radius $d R$ field is for the radial change in the Booster at extraction if known, otherwise the default is zero. The calculated extraction energy can then be used to calculate the NSRL energy loss.

Note that the dEdx, MeV/n, Beta, and Gamma are the parameters for the beam upon exit of the material. 
Appendix C: NSRL Target Room Layout
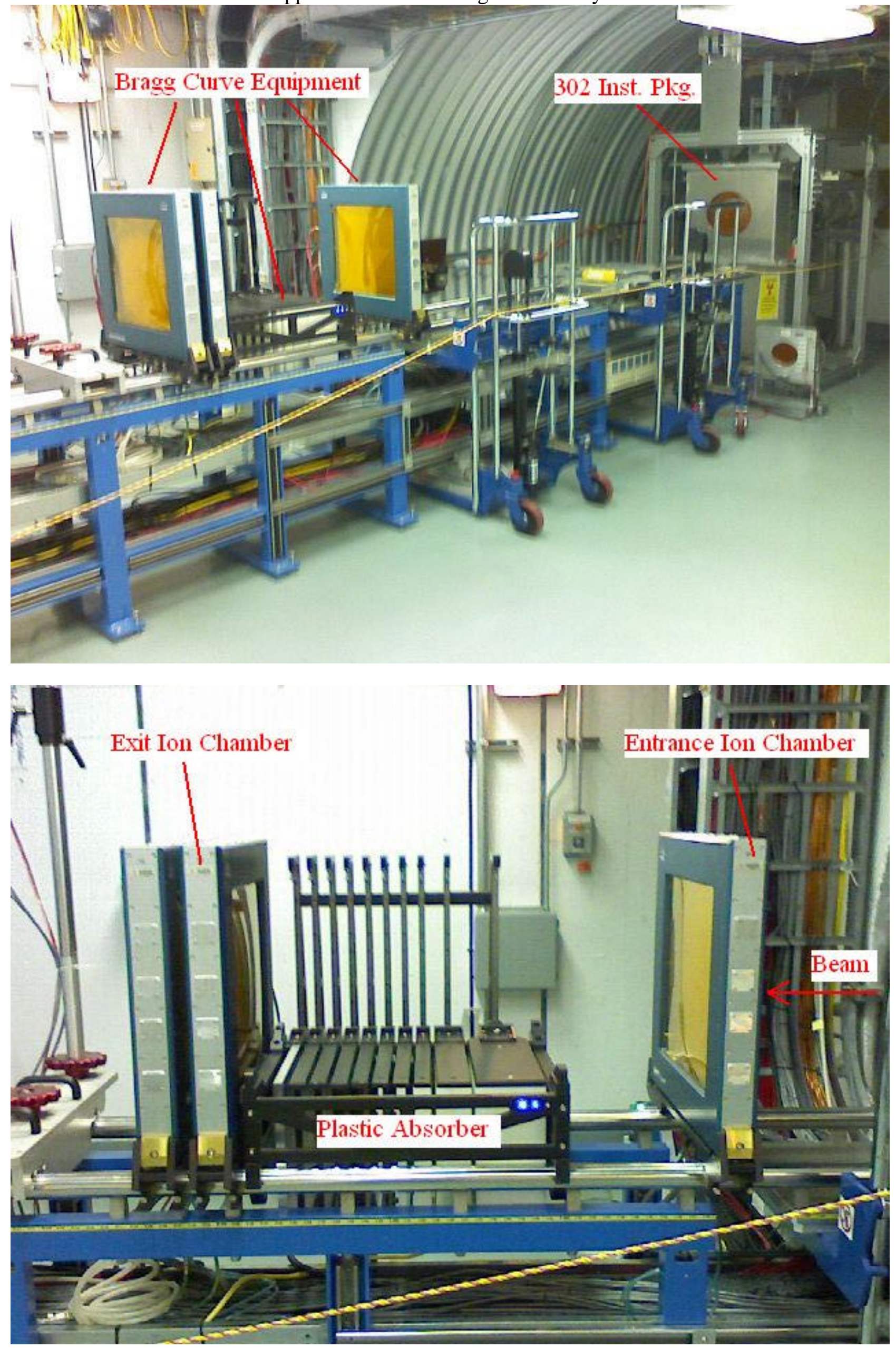
Appendix D: Instrument Package Cross Section

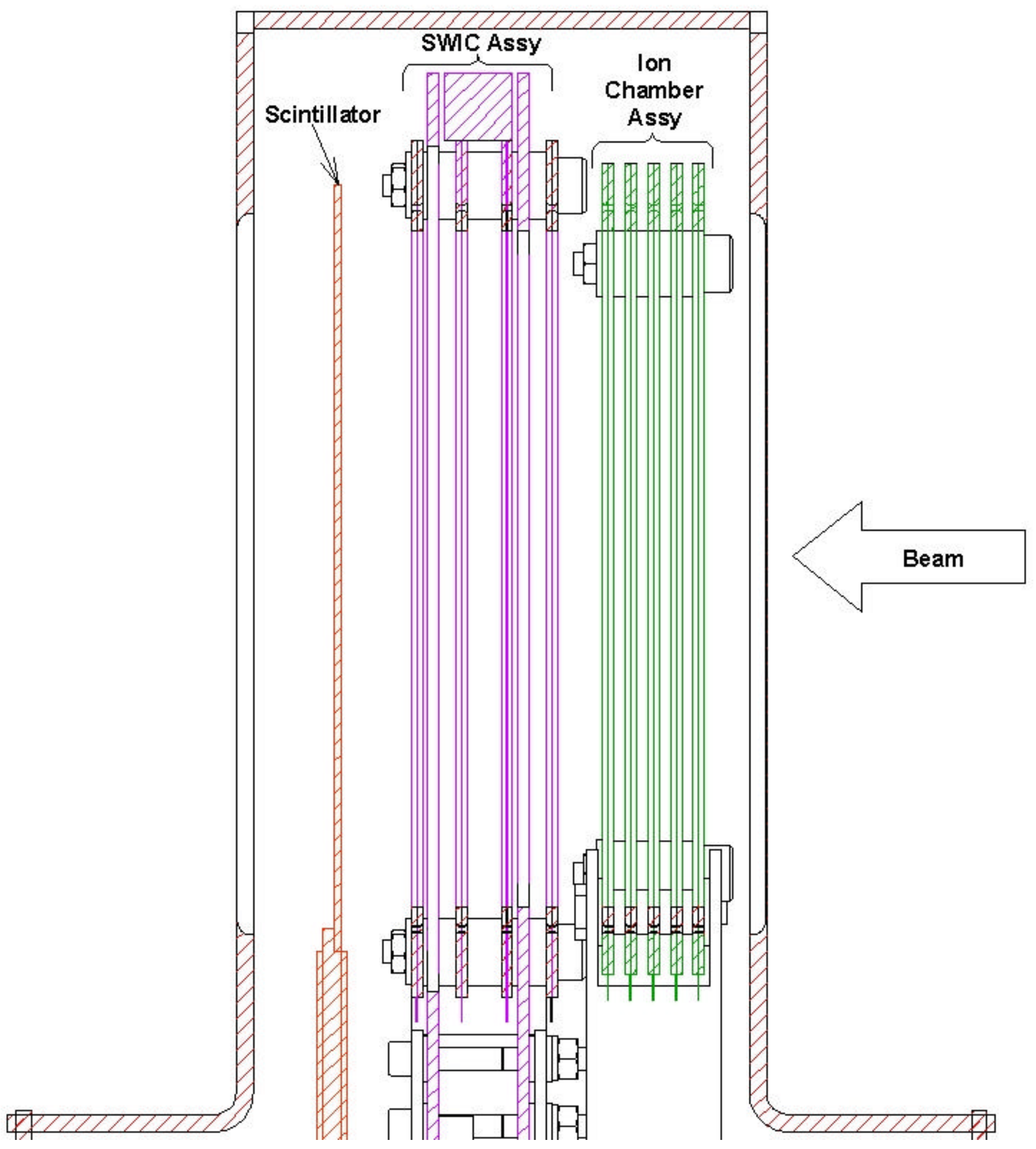




\section{References}

[1] Nuclear and Particle Physics, W.S.C. Williams, pg 234-239

[2] An Empirical Model for the Response of BtA Multiwires to Different Ions, C-A/AP\#221, K. Zeno, October 2005

[3] Atomic Parameters, C. J. Gardner, located at http://www.cadops.bnl.gov/AGS/Operations/GardnerNotes/NsrlNotes/AtomicParameters.pdf

[4] Nsrl\# Schedule and Parameters, C. J. Gardner, located at http://www.cadops.bnl.gov/AGS/Operations/GardnerNotes/NsrlRuns/

[5] NIST Physics Laboratory, located at

http://physics.nist.gov/cgi-bin/Star/compos.pl?ap 